\title{
Are antiphospholipid antibodies just a common epiphenomenon or are they causative of immune-mediated coagulopathy in COVID-19?
}

\author{
Diana Castillo-Martínez $^{1} \cdot$ Zaira Torres $^{2} \cdot$ Luis M. Amezcua-Guerra $^{2,3}$ (D) $\cdot$ Carlos Pineda $^{4}$ (D) \\ Received: 4 February 2021 / Revised: 22 March 2021 / Accepted: 29 March 2021 / Published online: 7 April 2021 \\ (C) International League of Associations for Rheumatology (ILAR) 2021
}

\begin{abstract}
The coronavirus disease 2019 (COVID-19) is the largest public health emergency in recent times. A significant number of patients develop a severe form of COVID-19 characterized by coagulopathy, organ failure, and elevated mortality. In addition, an unusually high frequency of antiphospholipid antibodies (aPLs) has been found in patients with COVID-19. These clinical and serological manifestations closely resemble those seen in the antiphospholipid syndrome (APS), especially in its catastrophic form, suggesting a role of aPLs in immune-associated coagulopathy. However, government bodies such as the American Society of Hematology have spoken out against the systematic search for aPLs in patients with COVID-19. In an attempt to bridge the gap on this hot topic, we conducted a comprehensive review of currently available cohort studies and case series systematically evaluating aPLs in COVID-19 patients. In this Perspective, we seek to identify both the frequency and the type of aPLs found in patients with COVID-19, as well as the potential association of these aPLs with vascular thrombosis and other distinctive characteristics of COVID-19. Furthermore, we investigated whether there is evidence that allows us to define the occurrence of aPLs in COVID-19 as an epiphenomenon, as has been observed in other systemic viral infections, or as antibodies against selfantigens bearing hallmarks that suggest a pathogenic role in immune-mediated thrombosis. Defining whether aPLs represent an epiphenomenon or they are actually involved in hemostatic abnormalities of COVID-19 is crucial both for uncovering novel mechanisms of immune-mediated thrombosis and for identifying potential prognostic biomarkers in this devastating disease.
\end{abstract}

Keywords Antiphospholipid antibodies $\cdot$ COVID-19 $\cdot$ Inflammation $\cdot$ Thrombosis

In December 2019, an outbreak of severe acute respiratory syndrome coronavirus 2 (SARS-CoV-2) infection occurred

Diana Castillo-Martínez and Zaira Torres participated equally; the order is alphabetical.

Luis M. Amezcua-Guerra

lmamezcuag@gmail.com

1 Department of Dermatology, Hospital General de Zona 32 Dr Mario Madrazo Navarro, Instituto Mexicano del Seguro Social, Mexico City Mexico

2 Department of Immunology, Instituto Nacional de Cardiología Ignacio Chávez, Juan Badiano 1, Sección XVI, 14080 Tlalpan Mexico City Mexico

3 Department of Health Care, Universidad Autónoma Metropolitana Xochimilco, Mexico City Mexico

4 Division of Musculoskeletal and Rheumatic Diseases, Instituto Nacional de Rehabilitación Luis Guillermo Ibarra Ibarra, Mexico City Mexico in Wuhan, China, and rapidly spread worldwide. The World Health Organization (WHO) officially named the disease as coronavirus disease 2019 (COVID-19), which is now considered to be the largest public health emergency in the last century. Although most patients with COVID-19 are asymptomatic or develop only a mild condition, a significant proportion of patients develop a severe form of the disease, which is featured by pneumonia, coagulopathy, hyperinflammation, and organ failure [1].

On April 8, 2020, Zhang et al. described three severely ill COVID-19 patients with multiple cerebral infarctions who had circulating anticardiolipin (aCL) and anti- $\beta 2$ glycoprotein I (a $32 \mathrm{GPI})$ antibodies, suggesting for the first time that coagulopathy associated with COVID-19 could be an acquired thrombophilia close to the spectrum of antiphospholipid syndrome (APS) [2]. However, a short time later, the American Society of Hematology (ASH) performed an antiphospholipid antibody (aPL) screen in 27 patients with COVID-19, in which they found that only four patients had 
lupus anticoagulant (LA) [3]. In contrast, no patient was positive for aCL or aß2GPI antibodies. Based on the wellrecognized fact that aPLs may transiently arise during acute infection, inflammation, or thrombosis, the ASH strongly recommended against routine aPL testing in COVID-19 patients, unless clinically indicated by the history or as part of a research protocol [3]. On the other hand, a higher-thanexpected number of thrombotic episodes have been reported, both venous (pulmonary thromboembolism, venous sinus thrombosis, deep vein thrombosis) and arterial (myocardial infarction and stroke) in patients with COVID-19, despite the use of prophylactic or therapeutic anticoagulation [4]. As a growing body of evidence on the frequency and clinical associations of aPLs in COVID-19 is emerging, this issue has become a hot topic in clinical (rheumatology) practice.

A first question arises: do circulating levels of aPLs increase in COVID-19 patients? Currently, there are approximately 20 manuscripts with a suitable methodological design (case series and cohort studies) that address this issue. Table 1 summarizes the main data of these studies. Overall, the frequency of circulating aPL in COVID-19 patients has been consistently high, with figures around 54\%, although the frequency between studies varies widely, ranging from 8 to $96 \%$ $[5,6]$. This heterogenicity may reflect differences between the clinical phenotype of the patients studied (mild versus severe disease and early versus late disease), the type of aPL evaluated ("criteria" versus "non-criteria" antibodies) [7], or the presence of disease complications (i.e., venous and pulmonary thromboembolism or stroke). The role of ethnic, genetic, and geographic background cannot be ruled out.

The variety of aPLs described in COVID-19 is interesting. Most studies have focused on testing "criteria" antibodies. A positive test for LA is found in approximately one of every two patients with COVID-19, while the presence of aCL and aß2GPI antibodies has been observed less frequently (around $10 \%$ for each) [8]. Concerning "non-criteria" antibodies, it should be noted that a wide variety of aPLs have been described in COVID-19 [7]. These include antiphosphatidylserine (aPS), anti-prothrombin (aPT), and antiannexin $\mathrm{V}(\mathrm{aAnnV})$ antibodies in both IgG and IgM isotypes, as well as aCL and aß2GPI in IgA isotypes [6, 9-13]. Both the high frequency and diversity of aPLs strongly suggest that these antibodies are actively induced during acute SARSCoV-2 infection. It is noteworthy that this prevalence is similar to that observed in several autoimmune diseases, although less than that found in patients with primary APS [14].

Once the elevated prevalence of aPL in COVID-19 has been established, the next issue to answer is: are these aPLs associated with the development of vascular thrombosis, or at least these antibodies are present in a specific clinical setting? Studies have consistently shown that aPLs occur in patients with COVID-19-associated hyperinflammation [15], a condition characterized by unusually high levels of C-reactive protein, ferritin, D-dimer, and interleukin-6, as well as increased activity of neutrophils $[10,16]$. In parallel, aPLs are found more frequently in patients with severe or critical illness than in their counterparts with a milder disease $[11,17]$. During acute SARS-CoV-2 infection, a consistent association has been observed between aPL positivity and the presence of other acquired thrombophilias, including protein $\mathrm{C}$, protein $\mathrm{S}$, antithrombin, and factor XII deficiency [12, 18, 19]. Therefore, low activity of natural anticoagulants and the presence of aPLs together may contribute to COVID-19 coagulopathy, although a pathogenic link remains to be demonstrated.

After the aforementioned association between circulating aPLs and the occurrence of stroke [2], other authors have explored the prothrombotic effects of these antibodies, reporting contradictory results. Several studies have consistently shown that aPLs are associated with the development of vascular thrombosis, particularly pulmonary thromboembolism and stroke $[6,9,11,12,16,20,21]$. A recent study has suggested that aPLs, even in weak or transient titers, are commonly found in patients hospitalized for COVID-19 [22]. This study identified that aPLs significantly associated with the occurrence of thrombotic events are $\mathrm{aCL}$ in $\mathrm{IgG}$ and $\mathrm{IgM}$ isotypes, a $32 \mathrm{GPI}$ in IgA isotype, and positive LA. Furthermore, the triple positivity of aPLs seems to be of special relevance [22]. There are even some studies suggesting that the presence of aPLs may be a poor prognostic marker, as they are positive in COVID-19 patients who will eventually have a torpid clinical course $[10,11,17,23]$. In contrast, other studies have failed to demonstrate an association between the presence of aPLs and the occurrence of thrombosis of any type $[5,13,14,17,24,25]$.

Infection-induced aPLs are typically transient and nonpathogenic. In SARS-CoV-2 infection, the concurrence of aPLs, large-vessel thromboses, thrombotic microangiopathy, and livedoid eruptions with complement activation over a short period of time strongly suggests an active role for these antibodies, leading to a condition that closely resembles catastrophic APS [16, 26]. Although the presence of LA in COVID-19 has been suggested to be spurious as most patients are on anticoagulant therapy, the use of diluted Russell's viper venom time (dRVVT) assays containing heparinase, which neutralizes any effect of heparins, virtually eliminates the possibility of false positive detection for LA [19]. Furthermore, the presence of aPS and aPT antibodies, which are associated with a prolonged activated partial thromboplastin time (aPTT), supports that LA positivity is due to circulating inhibitors in COVID-19 (i.e., autoantibodies against phospholipidprotein complexes) [9-11, 13, 24, 27]. Simultaneous elevations of anti-SARS-CoV-2 IgA, aCL IgA, and aß2GPI IgA antibodies in patients with severe COVID-19, but not in those with mild disease, suggest that a vigorous antiviral IgA response, possibly triggered in the bronchial mucosa, may 


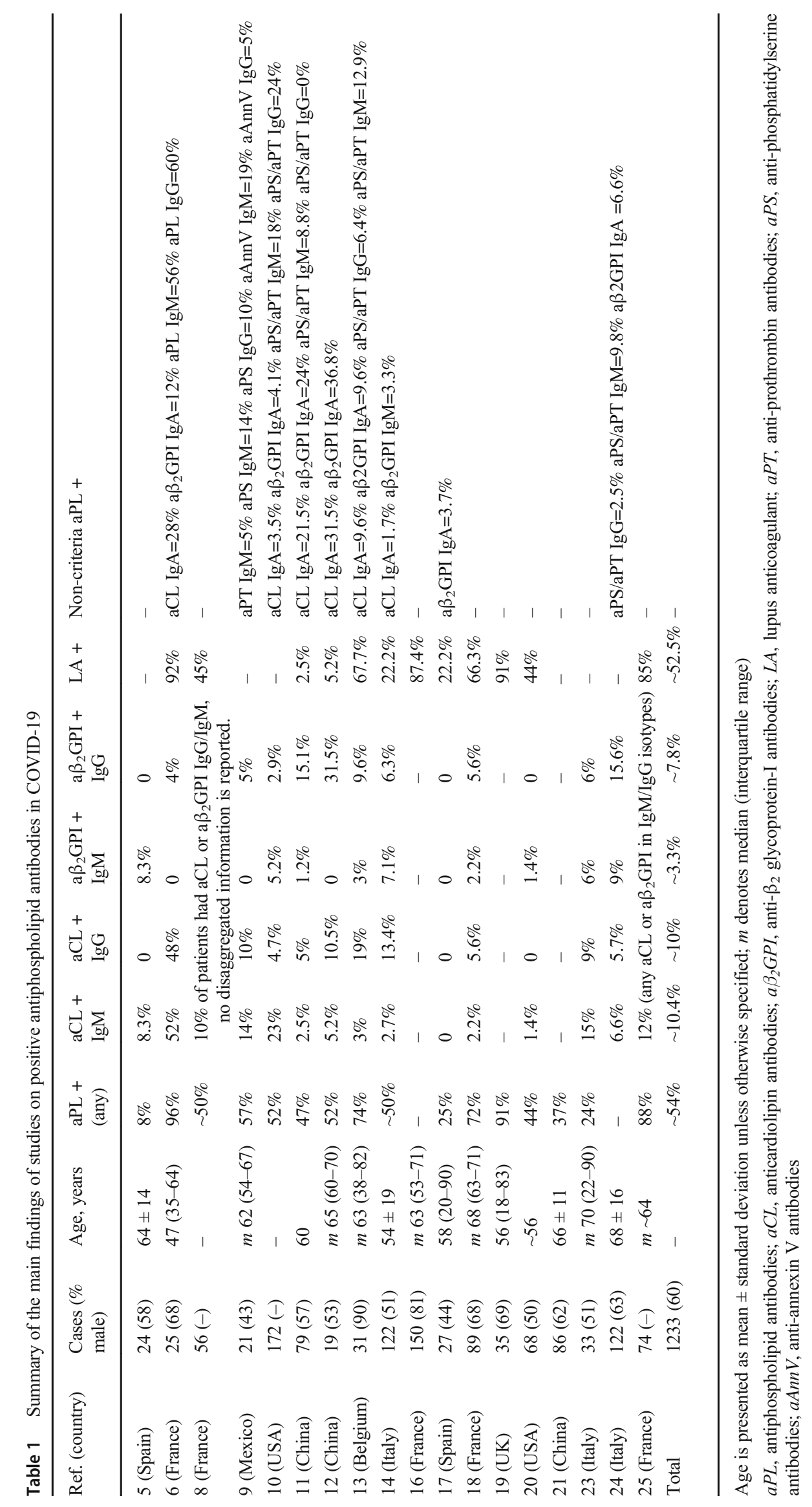


induce systemic autoimmunity [28]. Epitopic characterization of a $32 \mathrm{GPI}$ antibodies recently showed that reactivity against the $\mathrm{N}$-terminal domain 1 (anti-D1) or the $\mathrm{C}$-terminal domains 4-5 (anti-D4-5) is found in approximately 5\% (for each of the reactivities) of COVID-19 patients with positive aß2GPI antibodies [24]. Although anti-D1 antibodies are characteristically associated with the development of thrombosis in APS patients, anti-D4-5 antibodies are often described in asymptomatic aPL carriers [29], suggesting the possibility of the simultaneous induction of both pathogenic and nonpathogenic aPLs in COVID-19, which would require additional prothrombotic stimuli to facilitate the development of thrombosis. This is in line with the "two-hit" hypothesis of vascular APS causality [30].

Finally, do these aPLs increase transiently, as in different inflammatory-mediated conditions, or do they remain high enough to meet current guidelines for classifying APS [31]? This is a pending issue that requires further investigation [32]. No study yet includes confirmatory tests for aPL at 12 weeks, which is the standardized time frame for classifying APS [31]. In patients with APS, aPL tests remain positive for long periods, whereas epiphenomenon-induced aPLs, on the other hand, may be transient. A single study reported retesting in ten patients with COVID-19 who initially had positive LA, showing that nine of them tested negative 1 month after their first positive result [13].

Defining whether aPLs represent an epiphenomenon or they are actually involved in hemostatic abnormalities of COVID-19 is crucial both for uncovering novel mechanisms of immune-mediated thrombosis and for identifying potential prognostic biomarkers in this devastating disease.

Data availability Not applicable.

\section{Declaration}

Ethics approval Not applicable.

Consent to participate Not applicable.

Consent for publication Not applicable.

Disclosures None.

\section{References}

1. Wang D, Hu B, Hu C et al (2020) Clinical characteristics of 138 hospitalized patients with 2019 novel coronavirus-infected pneumonia in Wuhan, China. JAMA 323:1061-1069

2. Zhang Y, Xiao M, Zhang S et al (2020) Coagulopathy and antiphospholipid antibodies in patients with Covid-19. N Engl J Med 382:e38

3. https://www.hematology.org/covid-19/covid-19-and-apl-ab. Accessed 7 Dec 2020
4. Ahmed S, Zimba O, Gasparyan AY (2020) Thrombosis in coronavirus disease 2019 (COVID-19) through the prism of Virchow's triad. Clin Rheumatol 39:2529-2543

5. Galeano-Valle F, Oblitas CM, Ferreiro-Mazón MM et al (2020) Antiphospholipid antibodies are not elevated in patients with severe COVID-19 pneumonia and venous thromboembolism. Thromb Res 192:113-115

6. Pineton de Chambrun M, Frere C et al (2020) High frequency of antiphospholipid antibodies in critically ill COVID-19 patients: A link with hypercoagulability? J Intern Med. https://doi.org/10.1111/ joim. 13126

7. Funke A, Staub HL, Monticielo OA et al (2020) Non-criteria antiphospholipid antibodies: A narrative review. Rev Assoc Med Bras 66:1595-1601

8. Harzallah I, Debliquis A, Drénou B (2020) Lupus anticoagulant is frequent in patients with Covid-19. J Thromb Haemost 18:2064 2065

9. Amezcua-Guerra LM, Rojas-Velasco G, Brianza-Padilla M et al (2020) Presence of antiphospholipid antibodies in COVID-19: Case series study. Ann Rheum Dis. https://doi.org/10.1136/ annrheumdis-2020-218100

10. Zuo Y, Estes SK, Ali RA et al (2020) Prothrombotic autoantibodies in serum from patients hospitalized with COVID-19. Sci Transl Med. https://doi.org/10.1126/scitranslmed.abd3876

11. Xiao M, Zhang Y, Zhang S et al (2020) Antiphospholipid antibodies in critically ill patients with COVID-19. Arthritis Rheum 72: 1998-2004

12. Zhang Y, Cao W, Jiang W et al (2020) Profile of natural anticoagulant, coagulant factor and anti-phospholipid antibody in critically ill COVID-19 patients. J Thromb Thrombolysis 50:580-586

13. Devreese KMJ, Linskens EA, Benoit D, Peperstraete H (2020) Antiphospholipid antibodies in patients with COVID-19: A relevant observation? J Thromb Haemost 18:2191-2201

14. Gatto M, Perricone C, Tonello M et al (2020) Frequency and clinical correlates of antiphospholipid antibodies arising in patients with SARS-CoV-2 infection: Findings from a multicentre study on 122 cases. Clin Exp Rheumatol 38:754-759

15. Amezcua-Guerra LM (2020) Brief annotations on cytokine release syndrome and interleukin-6 therapeutic blockage in SARS-CoV-2/ COVID-19. Arch Cardiol Mex 90(Supl):84-87

16. Helms J, Tacquard C, Severac F et al (2020) High risk of thrombosis in patients with severe SARS-CoV-2 infection: A multicenter prospective cohort study. Intensive Care Med 46:1089-1098

17. Gutiérrez López de Ocáriz X, Castro Quismondo N, Vera Guerrero E, Rodríguez Rodríguez M, Ayala Díaz R, Martínez López J (2020) Thrombosis and antiphospholipid antibodies in patients with SARS-COV-2 infection (COVID-19). Int J Lab Hematol 42: e280-e282

18. Ferrari E, Sartre B, Squara F et al (2020) High prevalence of acquired thrombophilia without prognosis value in patients with coronavirus disease 2019. J Am Heart Assoc 9:e017773

19. Bowles L, Platton S, Yartey N et al (2020) Lupus anticoagulant and abnormal coagulation tests in patients with Covid-19. N Engl J Med 383:288-290

20. Reyes Gil M, Barouqa M, Szymanski J, Gonzalez-Lugo JD, Rahman S, Billett HH (2020) Assessment of lupus anticoagulant positivity in patients with coronavirus disease 2019 (COVID-19). JAMA Netw Open 3:e2017539

21. Fan S, Xiao M, Han F et al (2020) Neurological manifestations in critically ill patients with COVID-19: A retrospective study. Front Neurol 11:806

22. Le Joncour A, Frere C, Martin-Toutain I et al (2021) Antiphospholipid antibodies and thrombotic events in COVID-19 patients hospitalized in medicine ward. Autoimmun Rev 20: 102719 
23. Pascolini S, Vannini A, Deleonardi G et al (2020) COVID-19 and immunological dysregulation: Can autoantibodies be useful? Clin Transl Sci. https://doi.org/10.1111/cts.12908

24. Borghi MO, Beltagy A, Garrafa E et al (2020) Anti-phospholipid antibodies in COVID-19 are different from those detectable in the anti-phospholipid syndrome. Front Immunol 11:584241

25. Siguret V, Voicu S, Neuwirth M et al (2020) Are antiphospholipid antibodies associated with thrombotic complications in critically ill COVID-19 patients? Thromb Res 195:74-76

26. Magro C, Mulvey JJ, Berlin D et al (2020) Complement associated microvascular injury and thrombosis in the pathogenesis of severe COVID-19 infection: A report of five cases. Transl Res 220:1-13

27. Pengo V, Del Ross T, Ruffatti A et al (2018) Lupus anticoagulant identifies two distinct groups of patients with different antibody patterns. Thromb Res 172:172-178

28. Hasan Ali O, Bomze D, Risch L et al (2020) COVID-19 is associated with elevated serum IgA and antiphospholipid IgA-antibodies. Clin Infect Dis. https://doi.org/10.1093/cid/ciaa1496

29. Andreoli L, Chighizola CB, Nalli C et al (2015) Clinical characterization of antiphospholipid syndrome by detection of IgG antibodies against $\beta 2$-glycoprotein I domain 1 and domain 4/5: Ratio of anti-domain 1 to anti-domain $4 / 5$ as a useful new biomarker for antiphospholipid syndrome. Arthritis Rheum 67:2196-2204

30. Meroni PL, Raschi E, Grossi C et al (2012) Obstetric and vascular APS: Same autoantibodies but different diseases? Lupus 21:708710

31. Miyakis S, Lockshin MD, Atsumi T et al (2006) International consensus statement on an update of the classification criteria for definite antiphospholipid syndrome (APS). J Thromb Haemost 4:295306

32. Gkrouzman E, Barbhaiya M, Erkan D, Lockshin MD (2020) Reality check on antiphospholipid antibodies in COVID-19associated coagulopathy. Arthritis Rheum. https://doi.org/10. 1002/art.41472

Publisher's note Springer Nature remains neutral with regard to jurisdictional claims in published maps and institutional affiliations. 